STRUCTURAL SCIENCE CRYSTAL ENGINEERING MATERIALS

ISSN 2052-5206

Keywords: invariom modelling;

duplicate structures; metal-atom identification.

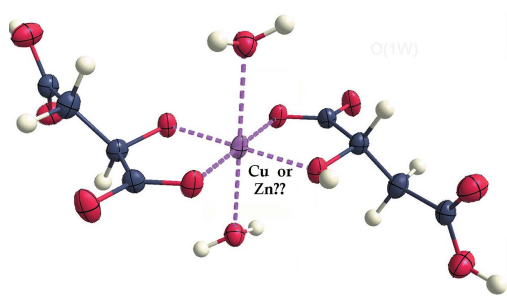

(C) 2017 International Union of Crystallography

\section{Ascertaining the correctness of the assigned central $3 d$ transition metal in coordination complexes}

\author{
Louis J. Farrugia*
}

School of Chemistry, University of Glasgow, Glasgow G12 8QQ, UK. *Correspondence e-mail:

louis.farrugia@glasgow.ac.uk

In their article, Birger Dittrich and co-workers (2017) describe an improved method of ascertaining the correctness or otherwise of the assigned central $3 d$ transition metal in coordination complexes, based solely on the diffraction data. As the authors imply, this would be a potentially very useful tool in the arsenal of crystallographic reviewers in cases where there may be some doubt about this. This in turn leads onto its potential use in detecting fraudulent publications or journal submissions - more on this issue later in this comment. Firstly I will attempt to explain the method they used to the interested non-specialist.

The central problem with relying solely on the diffraction data to make a distinction between two (or more) equally plausible central metal ions in coordination complexes is that the difference between the scattering power of such neighbouring atoms is not always sufficient to be decisive in least-squares refinements. This is because any errors in the scattering factor (i.e. identity of the element) in a crystallographic least-squares refinement could be subsumed into other refined parameters, such as the overall scale factor, or the displacement parameters, or indeed into both. There is likely to be a high correlation between the population of the metal atom (not normally but potentially refinable) and the above-mentioned parameters, making the refined magnitudes of these parameters somewhat suspect. Relatively poor quality and/or low-resolution diffraction data are likely to exacerbate this problem. Dittrich and co-workers propose using a more sophisticated model for the electron density of the atoms than the conventionally used spherical-atom model. In a nutshell, this more sophisticated model (the multipole model) makes allowances for the deformation of the atomic densities when chemical bonding is involved. It is a tried and tested methodology and has been successfully used for many years now. This multipole model requires many more parameters, which can only realistically be refined using highly accurate experimental structure factors. The novelty of the Dittrich approach is to use a library of aspherical scattering factors (obtained either theoretically or from experimental data) as fixed contributions, making it accessible for use with 'normal' quality low-resolution diffraction data.

There are several such libraries now available, but the types of chemical environments embedded in them are somewhat limited to relatively simple organic molecules containing only light atoms. Dittrich and co-workers get around this problem by performing a density functional theory calculation on the actual molecule under investigation, and extracting the aspherical scattering factors for all the elements in the coordination complex under investigation, in their various chemical environments, including the transition metals. When least-squares refinements are now undertaken using the 'normal' quality low-resolution diffraction data (but keeping the aspherical scattering parameters obtained by the quantum calculation fixed for all atoms) it has been shown that in most cases the distinction between neighbouring atoms becomes much amplified, compared with using a much simpler spherical atom scattering model. This in turn allows a much more decisive distinction to be made between two possible central atoms.

However, and this is made clear by the authors, such a methodology is far from being one which is straightforwardly implementable in an easy to use computer program and would still require considerable expertise to undertake such a calculation. Nevertheless, they did note that in many cases the distinction between two possible metals became much clearer even when just modelling the asphericity of the lighter atoms alone. Without the necessity of modelling the asphericity of the transition metal, their proce- 


\section{scientific commentaries}

dure would become considerably simplified. In any case, in its current implementation at least, the methodology of Dittrich and co-workers would be quite time-consuming and it is not at the stage where it could be used in automatic validation procedures such as checkCIF. The methodology will also have to be checked against more examples to ascertain its general applicability. It seems obvious that it will also be useful in other types of compounds of the $3 d$ transition metals such as organometallics, but how well will it work with heavier elements? These could include the other $d$-transition series, and more interestingly also the lanthanides, which would provide very demanding test cases.

I wish to say a few words about the second issue raised by this article, that of fraudulent publications. The existence of fraud in science is an insidious one, which seems to be becoming more frequent, though perhaps it is just that it is becoming easier to detect. The presence of fraud in the scientific arena tends to diminish the respect and prestige with which science is held in the public sphere, and may be contributing to the growth of anti-scientific sentiments. This is something that every conscientious scientist should be very concerned about. The authors of this paper have chosen to use, as test data sets for their methodology, 'duplicate structures' which had already been published and for which the diffraction data are in the public domain. As revealed by Dittrich and co-workers, the existence of incorrect structures, perhaps even fraud, may be possible to detect by this new method.

\section{References}

Wandtke, C. M., Weil, M., Simpson, J. \& Dittrich, B. (2017). Acta Cryst. B73, 794-804. 